\title{
Apport des études hydro-sédimentaires au projet d'aménagement d'un polder. (Ploubalay, Côtes d'Armor)
}

\author{
Chantal Bonnot-Courtois
}

Chargée de recherche CNRS

UMR 8586 PRODIG. Laboratoire de Géomorphologie et Environnement littoral. 15, bd de la mer 35800 DINARD.

\section{Résumé}

Dans le cadre de la réhabilitation et de l'aménagement du polder de Ploubalay (Côtes d'Armor), des études géomorphologiques, sédimentologiques et hydrologiques ont été menées afin de définir les possibilités de développer les potentialités de ce secteur. La fragilité de certaines parties de la digue, ancien ouvrage en terre, et l'analyse des niveaux de submersion soulignent les risques d'inondation de l'ensemble du polder. Une remise en eau partielle par création de mares peut être envisagée dans des conditions topographiques strictement définies afin de restaurer les caractéristiques écologiques de cette zone humide littorale.

Abstract

Geomorphological and hydro-sedimentological studies of the polder of Ploubalay (Côtes d'Armor) have been completed in order to rehabilitate the natural site. The high tide water levels and the weakness of the dyke lead to the inundation of the polder. In well defined topographical conditions, some areas could be partially open to seawater flooding in order to restore the ecology of this coastal wetland.

\section{Introduction}

Situé au fond de la baie de Lancieux dans le golfe de Saint-Malo, le polder de Ploubalay s'étend sur une surface de 70 ha et a été conquis sur les schorres depuis la fin du XVIIIème siècle, afin d'y développer des terrains à vocation axclusivement agricole (BRIAND, 1994). Actuellement, la majeure partie de cette zone a été rachetée par le Conservatoire du littoral qui souhaite assurer la sauvegarde et la mise en valeur du site qui possède des potentialités en terme d'accueil de l'avifaune et de restauration d'une zone humide littorale (Fig. 1). Une approche pluridisciplinaire (BONNOT-COURTOIS et al. 1999), associant le volet hydro-sédimentaire (présenté dans cet article) aux aspects botanique et ornithologique, a permis d'analyser l'état actuel du site et de mettre en évidence les caractéristiques physiques et écologiques du milieu afin de définir des mesures de réhabilitation et d'orienter des choix d'aménagement susceptibles de répondre à différentes options de gestion du polder. 


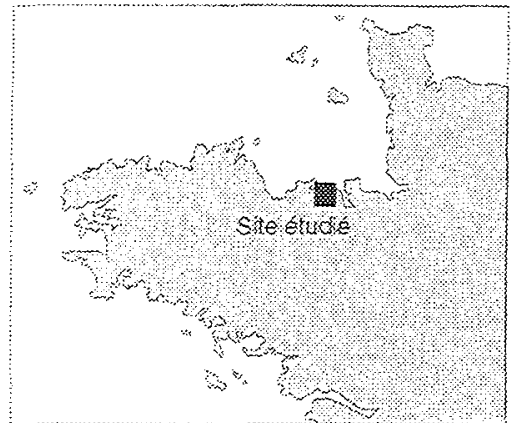

Fig. 1 : Description du polder et de l'étude des paramètres hydro-sédimentaires

Fig. 1 : Polder location and presentation of hydro-sedimentological studies
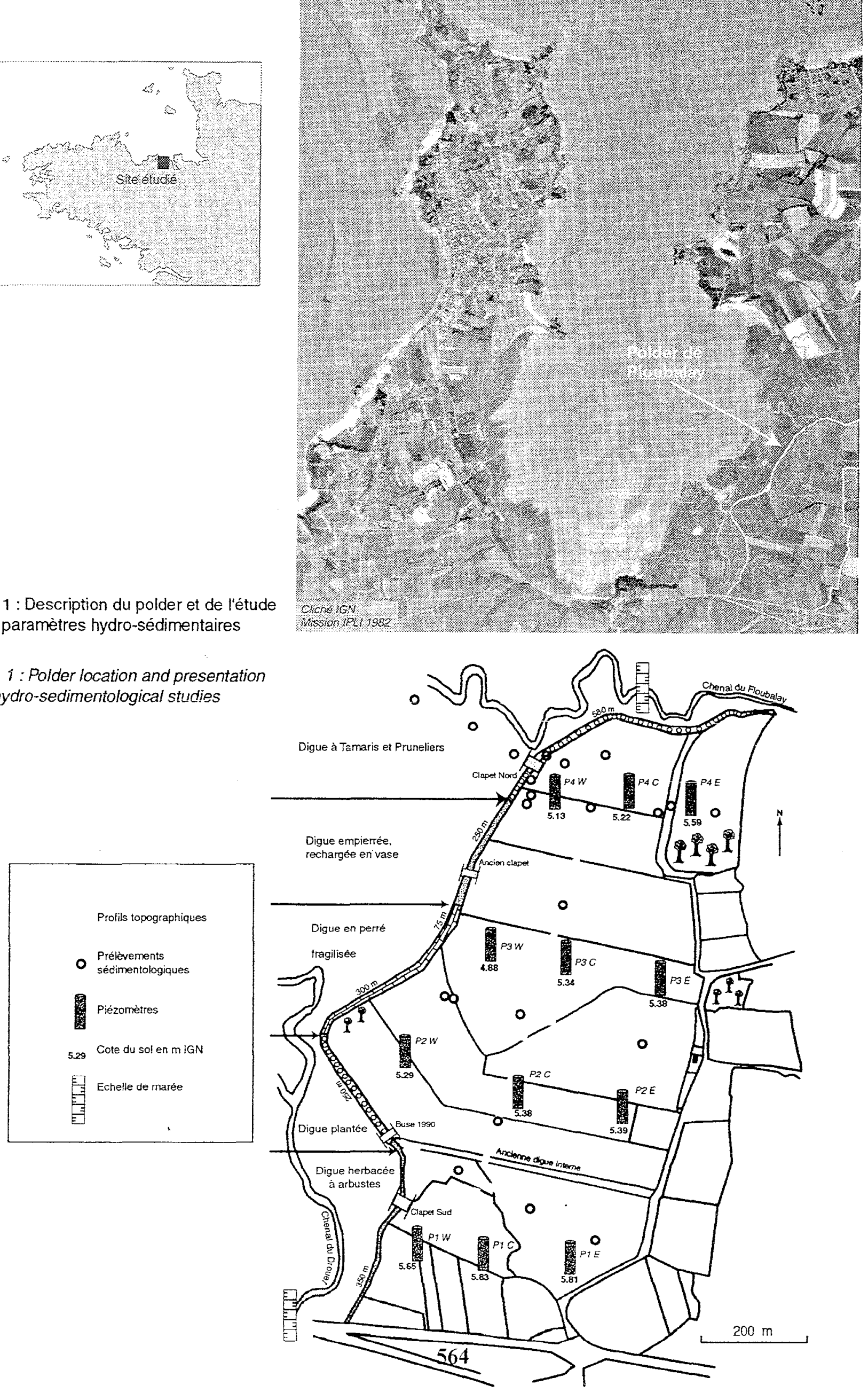


\section{Géomorphologie et topographie}

\subsection{Etat de la digue}

La digue qui borde le polder est une digue en terre, s'étendant sur un linéaire de $1800 \mathrm{~m}$ et qui se présente comme un talus partiellement empierré de forme parabolique de pente 2:1 côté mer et 1,5:1 côté polder (CHEVALLIER, 1996). Elle possède un bec central qui correspond au secteur de sollicitations maximales des courants et des houles et recouverte à cet endroit par un empierrement plus ou moins régulier qui protège le pied de digue. Les autres parties de la digue sont formées de vase, plus ou moins couverte de végétation parfois arbustive. L'état de l'ouvrage est très variable selon les secteurs (Fig. 1) :

* Dans la partie Sud, sur un linéaire de $350 \mathrm{~m}$, les terriers sont très nombreux et des brèches s'ouvrent régulièrement, laissant pénétrer l'eau de mer lors des marées de vive-eau. Au-delà, jusqu'au bec de la digue, la végétation arbustive bien développée et les terriers peu nombreux assurent une assez bonne tenue de la vase dans ce secteur peu exposé aux agents hydrodynamiques.

* La partie située à l'angle du bec de la digue est formée par un perré de pierres plus ou moins disjointes qui assurent une bonne protection de l'ouvrage côté mer. Le sommet de digue est cependant en mauvais état par affaissement de certaines zones où des brèches s'étaient creusées.

* Sur $250 \mathrm{~m}$ au Nord du secteur précédent, la digue constituée à l'origine par un perré, a subi de nombreux dégâts qui ont été réparés par des rechargements de vase, dont la tenue n'est pas bonne. Des morceaux de film plastique réapparaissent en de nombreux endroits sous un mélange de vase et de pierres et le colmatage des brèches a été effectué en prélevant la vase directement sur le haut schorre créant ainsi des affouillements en pied de digue. Les marques de fuagilité de l'ouvrage sont nettement visibles dans cette zone où des brèches se sont ouvertes sur plusieurs dizaines de mètres en février 1990, ce qui a provoqué l'inondation de toute la partie Nord du polder.

* Plus au Nord, la digue en vase est recouverte d'une abondante végétation arbustive mais des effondrements ont lieu, donnant naissance à des fissures longitudinales qui fragilisent le corps de digue. Le clapet installé au niveau d'un méandre du chenal du ruisseau du Floubalay, était constitué d'un système de tuyaux emboités et équipé d'une porte à flot. L'importance des courants à cet endroit à fragilisé l'ensemble du clapet qui a finalement été soulevé par la mer malgré de nombreuses réparations. Actuellement, le système fonctionne comme une buse sans clapet et constitue le seul point fonctionnel d'entrée et de sortie des eaux de la partie Nord du polder. 


\subsection{Topographie. Niveaux de submersion}

Des coupes topographiques transversales et longitudinale (Fig.1), raccordées au zéro IGN terrestre, ont été réalisées à l'intérieur du polder ainsi qu'un cheminement le long de la digue permettant d'avoir les cotes du haut schorre. En outre, les courbes de marée ont été établies côté mer pour différents coefficients au niveau des chenaux des deux ruisseaux qui encadrent le polder au Nord et au Sud (Fig. 1), et comparées aux niveaux atteints par l'eau dans le fossé de drainage interne dans les mêmes conditions de marée.

L'ensemble du polder est caractérisé par une topographie très plane, avec de faibles pentes dans deux directions, l'une de $0,16 \%$ d'Est en Ouest et l'autre du Sud vers le Nord de $0,14 \%$. La partie Est du polder se situe à une cote de $5 \mathrm{~m} 50$ IGN tandis que la partie occidentale, proche du chenal qui longe la digue est à environ $5 \mathrm{~m}$ IGN. Les chenaux et fossés de drainage qui traversent ou séparent les parcelles ont des profondeuris comprises entre $50 \mathrm{~cm}$ et $1 \mathrm{~m}$. Le haut estran est à $5 \mathrm{~m}$ IGN et le haut de la digue compris entre $7 \mathrm{~m} 20$ et $7 \mathrm{~m} 90 \mathrm{IGN}$, ce qui donne par rapport au zéro hydrographique des valeurs de $11 \mathrm{~m} 30$ pour le pied de digue et autour de $13 \mathrm{~m} 80 \mathrm{CM}$ pour le sommet de ia digue.

Les niveaux atteints par la marée de part et d'autre de la digue sont à mettre directement en relation avec la topographie du polder. Dans la partie centrale de celuici, le niveau moyen du haut schorre en pied de digue est à 5 m80 IGN69, et celui du polder est à $5 \mathrm{~m}$ IGN69 dans sa partie proximale. Les pleines mers de grande vive-eau mesurées à l'extérieur de l'exutoire atteignent une altitude de $7 \mathrm{~m} 05$ IGN, soit $2 \mathrm{~m}$ audessus du niveau du polder. Le problème prioritaire est donc celui de la solidité de la digue pour prévenir les risques d'inondation quasi-totale du polder. La description de l'ouvrage a montré ses zones de fragilité qui doivent impérativement être réparées.

Le seuil de la buse Nord se situe à $4 \mathrm{~m} 10 \mathrm{IGN}$ et le niveau maximum atteint par l'eau dans le fossé du polder a été de $4 \mathrm{~m} 40$ IGN pour la plus grande marée observée de coefficient 118. Dans la configuration actuelle de la buse Nord, le marnage maximum observé dans le canal périphérique interne est ainsi d'environ $30 \mathrm{~cm}$ seulement, ce qui va déterminer l'amplitude verticale des éventuels remodelages des berges.

\section{Hydrologie. Sédimentologie}

\subsection{Battement de la nappe et pénétration de l'eau de mer dans le polder}

Le suivi hydrologique de variation du niveau de la nappe phréatique a été réalisé à l'aide de 12 piézomètres répartis régulièrement sur l'ensemble du polder (Fig.1) et pour des conditions de marée très différentes. Sur la période d'observation de l'année 1998, aucune corrélation entre la hauteur de la marée et la hauteur de la nappe n'a pû être mise en évidence. Les jours où la nappe la plus haute a été observée (20 Janvier et 20 Avril 1998) correspondent à des périodes de marées de grande morte eau (coefficients 43 et 42 ). Les remontées du toit de la nappe sont en relation étroite avec 
l'ampleur des précipitations (Fig.2) et elle se situe parfois à moins de $10 \mathrm{~cm}$ de la surface du sol en période de pluviométrie élevée (Janvier et Avril).

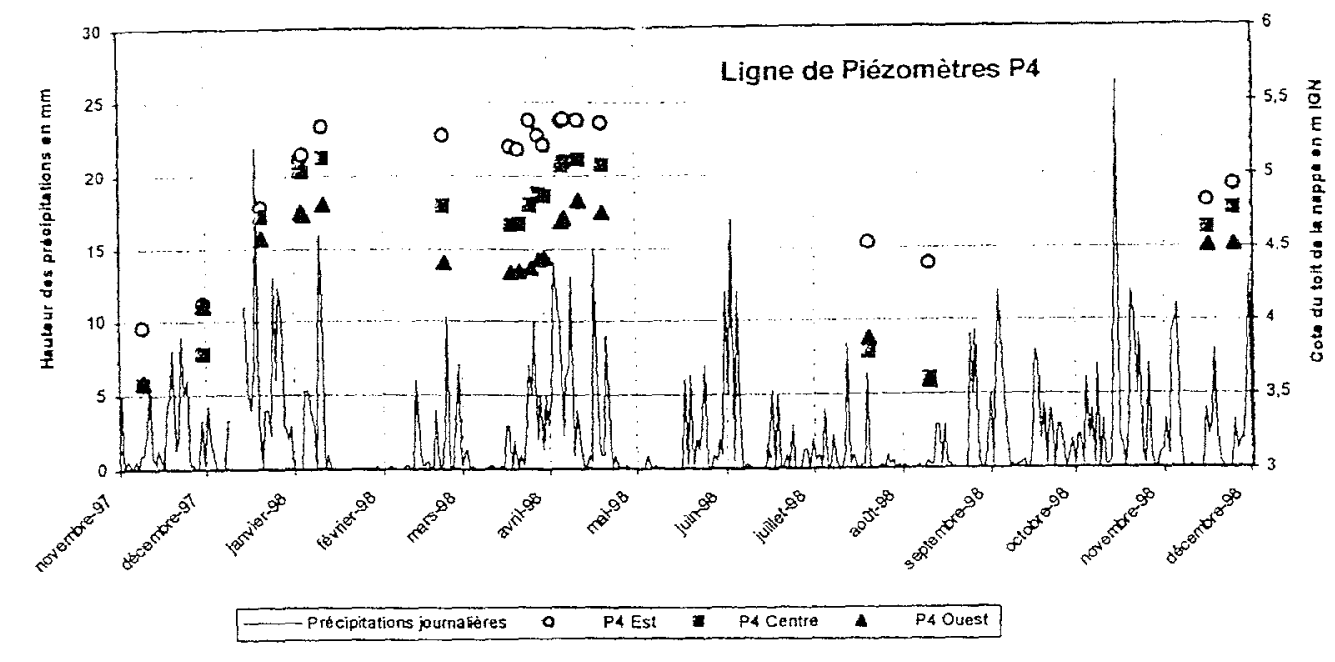

Fig. 2 : Evolution du toit de la nappe en fonction des précipitations en 1988.

Fig. 2 :Surface water table level variation with the rainfall during 1988

Les remontées d'eau salée suivies à l'intérieur du polder s'effectuent le long des chenaux principaux dans lesquels on observe une stratification haline, même sur une faible épaisseur de la tranche d'eau $(50 \mathrm{~cm})$. Les fossés de la partie Est du polder contiennent de l'eau douce et ne sont pas atteints par les plus fortes marées (Fig. 3). L'eau de surface de la nappe enregistre des variations de salinité beaucoup moins contrastées que dans les chenaux libres et de façon notable seulement dans certains secteurs qui coincident avec la proximité des chenaux actuels ou de l'ancien réseau de chenaux, figuré sur la carte des Ingénieurs Géographes de 1776. Cette répartition de ia salinité à l'intérieur du réseau hydrographique du polder dépend directement de l'amplitude de la marée, du niveau d'eau dans les chenaux au moment de l'observation et du régime pluviométrique qui détermine les apports d'eau douce susceptibles de diluer les eaux marines.

\subsection{Analyses sédimentologiques}

Une reconnaissance de la composition granulométrique des sédiments du polder (sédiments superficiels et des sondages des piézomètres), de la digue et de l'estran proche (cf. localisation des prélèvements, Fig.1) montre que le sol superficiel du polder est formé d'une couche de terre grumeleuse, plastique ( 60 à $80 \%<40 \mu \mathrm{m}$ ) dont l'épaisseur varie de $50 \mathrm{~cm}$ à $1 \mathrm{~m}$. Plus en profondeur, on rencontre généralement soit un sable fin à très fin, plus ou moins argileux, soit une couche argileuse compacte intercalée entre des niveaux plus sableux. Des sédiments sableux fins, presque toujours gorgés d'eau, sont assez systématiquement présents à la base des sondages. Tous les échantillons sont des sables très fins ou des silts plus ou moins riches en argiles, avec 

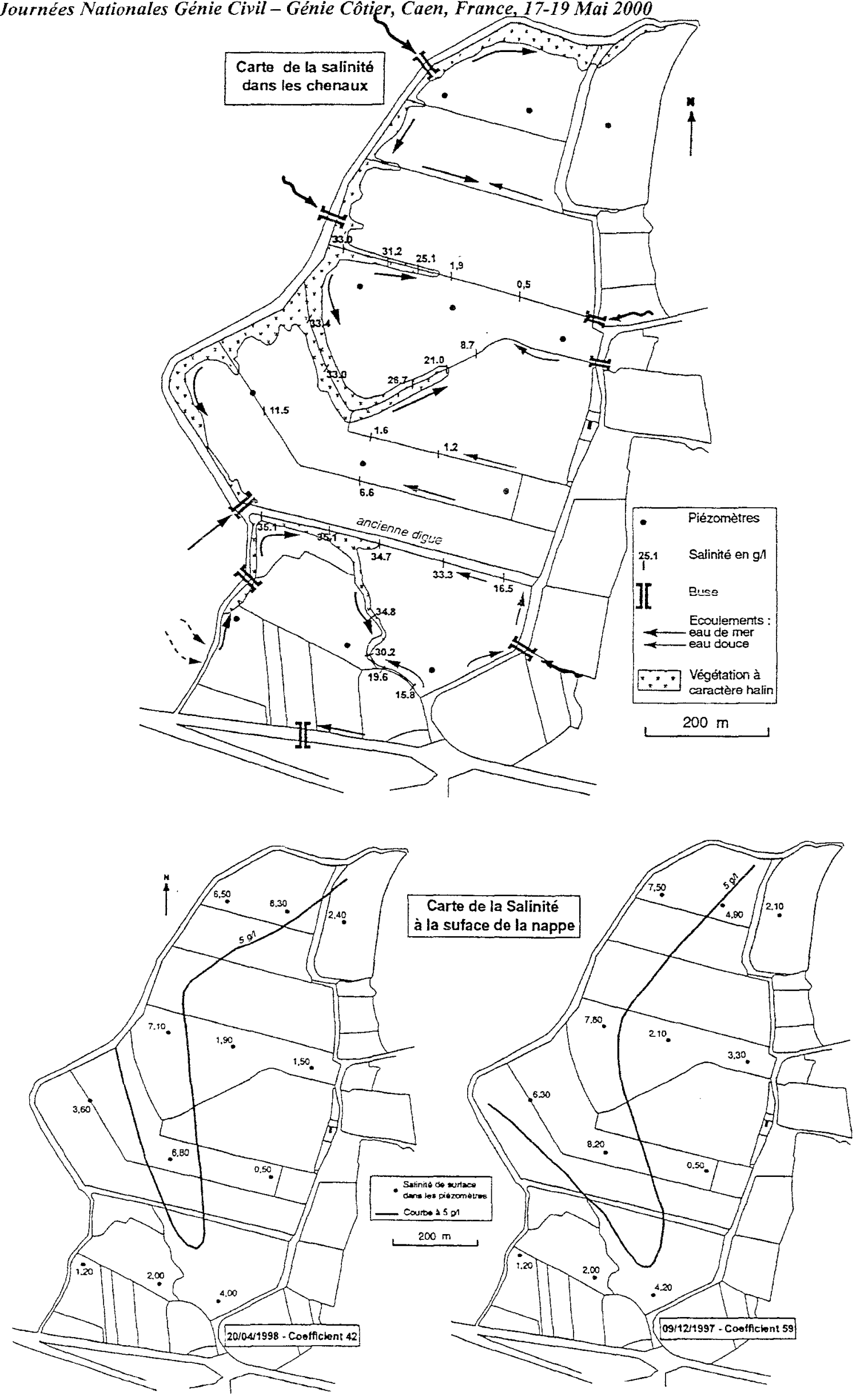

Fig. 3 : Répartition de la salinite dans le polder (chenaux et surface de la nappe) Fig. 3 : Salinity distribution in channels and superficial water table in the polder 
des courbes granulométriques plurimodales, le mode principal étant plus généralement situé dans les silts $(63 \mu \mathrm{m})$.

Les échantillons de la haute slikke sont des silts (médiane $=60 \mu \mathrm{m}$ ) qui contiennent une certaine proportion de vase (20 à 30\%). Les échantillons prélevés à différents niveaux du schorre sont formés de sédiments fins avec un mode dominant toujours inférieur à $40 \mu \mathrm{m}$. Les proportions de fraction fine sont importantes, comprises entre 40 et $75 \%$, quelle que soit la profondeur du prélèvement. Le sédiment prélevé à différents niveaux de la digue elle-même a une granulométrie comparable à celle des vases du schorre, avec une médiane inférieure à $40 \mu \mathrm{m}$ et des proportions de fraction fine dépassant la moitié du sédiment. Pour tous ces échantillons, un mode secondaire à $63 \mu \mathrm{m}$, donc silteux, s'associe à la fraction fine. Les éboulis en pied de digue, du côté du polder, contiennent $65 \%$ de fraction fine et le sédiment prélevé sur les berges du chenal du fossé de drainage interne est très vaseux $(70 \%$ de fraction $<40 \mu \mathrm{m})$.

Les sédiments extraits en baie de Lancieux en septembre 1998 et rapportés à proximité du clapet Nord pour restaurer la face interne du pied de digue sont beaucoup moins riches en fraction fine que ceux du schorre ou de la digue. Ils contiennent plus de $90 \%$ de fraction grossière supérieure à $40 \mu \mathrm{m}$ et sont formés de sables très fins avec un mode principal et unique à $80 \mu \mathrm{m}$ et une médiane à $70 \mu \mathrm{m}$. Ces sables très fins, silteux, sont donc très différents des vases de la digue ou du schorre externe, et leur cohésion est certainement moins bonne ce qui ne favorise pas la tenue de ces sédiments vis à vis de l'agitation. Les rechargements de la digue devront être effectués avec des matériaux moins sableux, plus résistants à l'érosion.

\section{Conclusion. Propositions d'aménagements}

Les résultats obtenus sur le site conduisent à envisager les futurs aménagements en fonction des caractéristiques physiques et écologiques du milieu qui sont déterminantes dans les choix de mise en valeur du polder (Fig.4).

Le suivi hydrologique des piézomètres a montré limpontance déterminante de la pluviométrie dans le battement de la nappe du polder. Sur la période d'observation de l'année 1998, les remontées du toit de la nappe sont en relation étroite avec l'ampleur des précipitations et l'influence de la marée n'a pas été mise en évidence. L'alimentation en eau de mer de futures mares ne pourra donc être effective que par l'intermédiaire des eaux libres circulantes dans le canal périphérique et le chenal majeur de la partie centrale du polder. Les cartes de répartition de la salinité ont bien montré que l'intrusion saline a lieu selon une bande orientée vers le Sud-Est et localisée dans zone du chenal interne actuel et des anciens chenaux. Ce secteur où les conditions d'alimentation en eau salée sont réunies peut donc constituer l'emplacement de mares en élargissant le confluent entre le chenal central et le fossé périphérique contre la digue. De plus, la présence d'une couche argileuse peu perméable à environ $1 \mathrm{~m} 50$ de profondeur dans 
l'ensemble des sondages effectués sur le polder permet de penser qu'un niveau minimum d'eau sera maintenu dans la mare à cet endroit.

Le clapet de la buse Nord n'est plus fonctionnel et ne doit pas être réinstallé si l'on souhaite favoriser les entrées d'eau de mer. Dans la configuration actuelle de la buse Nord, le marnage maximum observé dans le canal périphérique interne est d'environ $30 \mathrm{~cm}$ seulement, ce qui va déterminer l'amplitude verticale des éventuels remodelages des berges. A travers cette fourchette, les surfaces à aménager dépendront évidemment des pentes choisies pour développer tel ou tel niveau d'accueil pour les plantes et/ou pour les oiseaux.

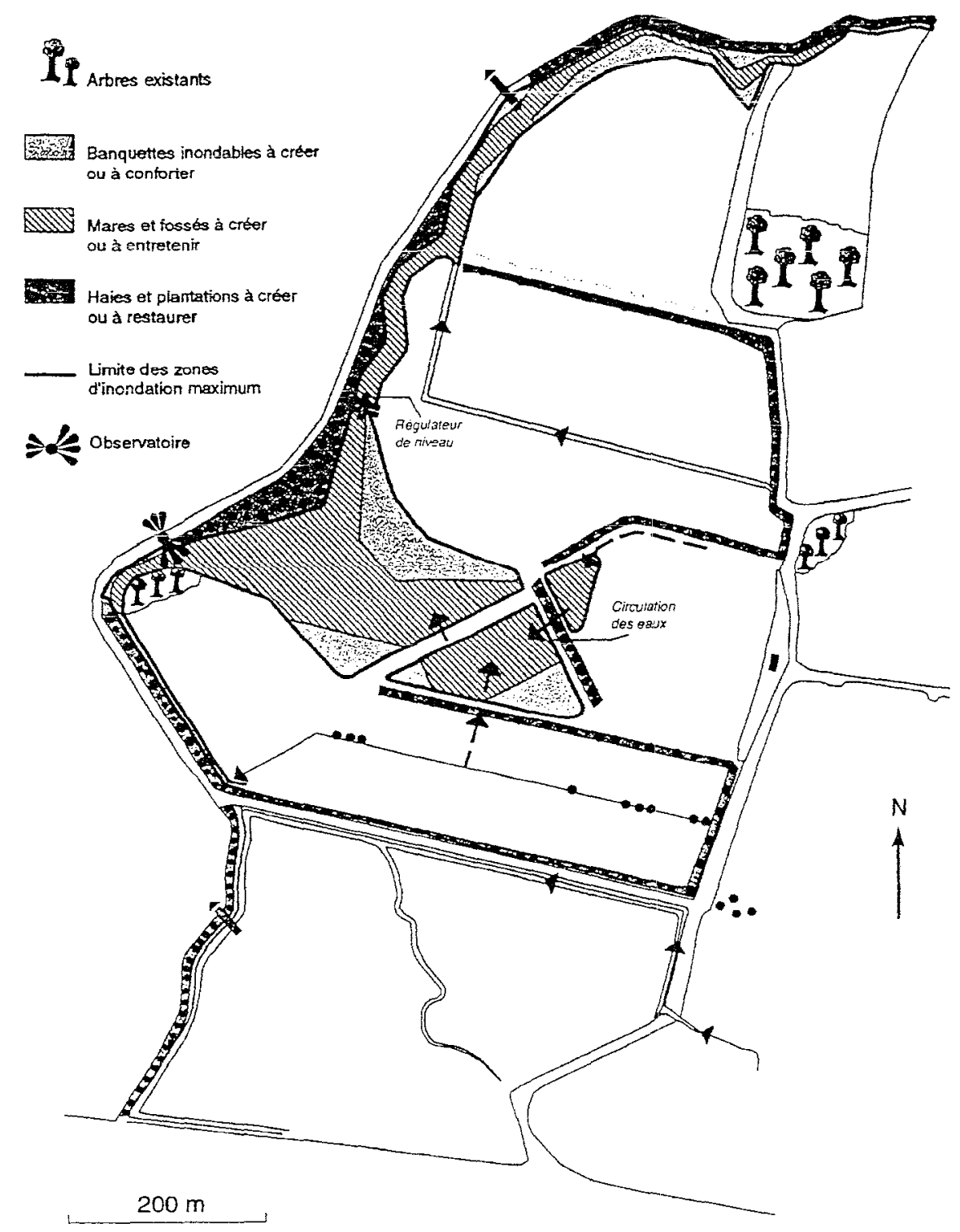

Fig. 4 : Propositions d'aménagement du polder Fig. 4 : Proposals for the polder development 
La création de plans d'eau permanents et l'élargissement du fossé interne nécessitent de faire pénétrer dans le polder des volumes d'eau de mer devant couvrir une surface environ 10 fois plus importante que celle des chenaux actuels. Le diamètre de la buse Nord devrait être augmenté pour pouvoir assurer des débits suffisants pour alimenter les plans d'eau salée, mais les contraintes de niveau de submersion doivent être précisément respectées. En effet, côté externe le seuil du radier (actuellement à $4 \mathrm{~m} 10 \mathrm{IGN}$ ) ne doit pas être trop profond dans le chenal du Floubalay afin d'éviter les entrées des eaux de début de flot, généralement très chargées en matières en suspension, et, côté interne, le niveau atteint par l'eau dans les fossés et les plans d'eau ne doit pas dépasser $5 \mathrm{~m}$ IGN qui correspond à la cote limite d'inondation du polder. Les banquettes inondables qui bordent les mares, situées entre $4 \mathrm{~m} 20$ et $4 \mathrm{~m} 50 \mathrm{IGN}$, joueront un rôle tampon entre la plan d'eau permanent ( $3 \mathrm{~m} 90 \mathrm{IGN}$ ) et le niveau du polder ( $5 \mathrm{~m}$ à $5 \mathrm{~m} 50 \mathrm{IGN})$.

En ce qui concerne la digue elle-même, les parties endommagées en perré doivent être reconstituées selon le modèle d'empierrement qui existe dejà et dont on a un exemple en très bon état près du bec de la digue. Le profil en travers, de forme trapézoïdale, des digues en perré existantes doit être conservé, avec une pente douce côté mer, et la restauration doit être effectuée à partir d'enrochements:maçonnerie en pierres sèches, de même nature que celles d'origine afin de conserver son caractère ancien à l'ouvrage. Les secteurs en terre doivent être rechargés selon les règles de tenue des talus, impliquant la nécessité d'une pente assez douce, et l'utilisation d'un matériau de rechargement dont la texture soit comparable à celle des vases déjà mises en place à lorigine de la digue. Les rechargements doivent être effectués avec une vase assez fine, peu sableuse dont la cohésion assure une bonne stabilité vis-à-vis des agents hydrodynamiques. La pente idéale peut être de $2: 1$ dans la mesure où une végétalisation de la surface est prévue. Cette revégétalisation est tout-à-fait nécessaire car elle consolide le talus exposé à l'agitation, mais elle doit être réalisée avec des espèces dont les racines ne déstabilisent pas le corps de l'ouvrage. Les vases extraites pour creuser les plans d'eau et élargir le fossé pourront être utilisées pour réparer la digue et la conforter sur son flanc interne de façon à éloigner le fossé du pied de digue.

La partie Sud, située entre l'ancienne digue et la route, fonctionne indépendemment du système Nord. L'altitude moyenne du marais est plus élevée ( $5 \mathrm{~m} 70$ IGN) et linfluence maritime y est réduite et limitée à l'angle Nord-Ouest du chenal actif. Cette zone devrait en principe être isolée des apports d'eau de mer car la buse Sud est équipée d'un clapet fonctionnel. Les entrées d'eau de mer s'effectuent par infiltrations au travers des nombreux terriers qui "minent" la digue dans ce secteur par ailleurs densément arboré. Si cette partie de l'ouvrage est réhabilitée et confortée, il ne devrait plus y avoir d'entrées incontrôlées d'eaux salées. 


\section{Références}

BONNOT-COURTOIS C. , LEVASSEUR J.E., MAHEO R. (1999) - Le polder de Ploubalay. Etude hydrosédimentaire, botanique et ornithologique du site. Projet de réhabilitation et d'aménagement. Rapport Conservatoire du Littoral, Plérin (22) / Lab. Géomorphologie Dinard, 125 pp.

BRIAND C. (1994) - Plan d'aménagement et de gestion du site du Tertre Corlieu à Lancieux (Côtes d'Armor). Extension du projet au polder de Ploubalay dans une perspective de développement d'un pôle Tourisme-Nature. Mémoire E.S.E.M., Orléans, 50 pp. + annexes.

CHEVALLIER M.L. (1996) - Mise en valeur du polder de Ploubalay (Côtes d'Armor). . Etude préliminaire. Rapport Conservatoire du Littoral, Plérin (22), 99 pp. + annexes.

Remerciements : Cette étude a bénéficié du financement du Conservatoire du Littoral de Plérn-Les Rosaires (Saint-Brieuc) avec le soutien du Conseil Général des Côtes d'Armor (Bureau des Espaces Naturels). 